\title{
Bivariate Interpolation of Potential Functions
}

\author{
By F. D. Burgoyne
}

1. Introduction. To solve Poisson's equation over a region, use is frequently made of a numerical method which evaluates the required function at the points of a rectangular grid. Usually, the error associated with such a process is of the fourth or sixth order in the various differences. The problem may then remain of evaluating the function at nongrid points. This can be done by bivariate interpolation. It is, however, possible to modify the usual formulae [1], [2], [3] so as to use fewer interpolation points, since Poisson's equation holds at each grid point. If, in addition, Laplace's equation holds, these modified formulae become quite simple and convenient to use. Such formulae are obtained by using a bivariate version of the "Mehrstellenverfahren" due to Collatz and others [4], [5], [6], [7]. These authors seek an accurate representation for a differential operator by means of a difference operator. They reduce the number of grid points needed for a given accuracy by assuming that the differential equation is approximately satisfied at each grid point. We use the same idea, but apply it to get better interpolation formulae. Of the very many such modified formulae we consider below two of the mid-point variety and one of the mid-panel variety, which have proved useful in practice. The error associated with these particular formulae is either of the fourth or of the sixth order, and is intended to match that of the method used to evaluate the function at the grid points. Higher order formulae, and formulae of other varieties, which may be required for special purposes, can be developed quite simply in the same manner. We consider also one cubature formula which has proved useful when integrating over a region throughout which Poisson's equation holds. This formula is a generalization of one due to Bickley [8].

2. Mid-Point Formulae. The commonly used bivariate Stirling interpolation formula with error of the $(n+1)$ th order may be written

$$
f_{\theta \phi}=\sum_{r=0}^{n} P_{r} f_{00},
$$

where

$$
\begin{aligned}
P_{2 s} & =\sum_{t=0}^{s} \theta_{2 s-2 t} \phi_{2 t} \delta_{x}{ }^{2 s-2 t} \delta_{y}{ }^{2 t}+\sum_{t=1}^{s} \theta_{2 s-2 t+1} \phi_{2 t-1} \mu_{x} \delta_{x}{ }^{2 s-2 t+1} \mu_{y} \delta_{y}{ }^{2 t-1}, \\
P_{2 s+1} & =\sum_{t=0}^{s} \theta_{2 s-2 t+1} \phi_{2 t} \mu_{x} \delta_{x}{ }^{2 s-2 t+1} \delta_{y}{ }^{2 t}+\sum_{t=0}^{s} \theta_{2 s-2} \phi_{2 t+1} \delta_{x}{ }^{2 s-2 t} \mu_{y} \delta_{y}{ }^{2 t+1},
\end{aligned}
$$

and we have written $\theta_{0}=1, \quad \theta_{1}=\theta, \quad \theta_{2}=\theta^{2} / 2 !, \quad \theta_{3}=\theta\left(\theta^{2}-1^{2}\right) / 3$ !, $\theta_{4}=\theta^{2}\left(\theta^{2}-1^{2}\right) / 4 !, \quad \theta_{5}=\theta\left(\theta^{2}-1^{2}\right)\left(\theta^{2}-2^{2}\right) / 5$ !, etc. It is usual to take $|\theta| \leqq \frac{1}{2}$, $|\boldsymbol{\phi}| \leqq \frac{1}{2}$. The error of (1) is approximately $P_{n+1} f_{00}$. If $f$ has continuous $(n+1)$ th

Received July 10, 1967. Revised September 18, 1967. 
order partial derivatives, an upper bound for the modulus of the error is $c_{n+1} M_{n+1} \max \left(h^{n+1}, k^{n+1}\right)$, where $c_{s}=\max _{\theta, \phi} \sum_{t=0}^{s}\left|\theta_{s-t} \phi_{t}\right|, \quad M_{s}$ is an upper bound for the modulus of all the sth order partial derivatives of $f$ in the region under consideration, and $h, k$ are the $x, y$ intervals. The formula involves the grid points numbered $0,1, \cdots, n$ in Fig. 1.

$\begin{array}{ccccccc} & & & 5 & & & \\ & & 4 & 3 & 4 & & \\ & 4 & 2 & 1 & 2 & 4 & \\ & 3 & 1 & 0 & 1 & 3 & 5 \\ & 4 & 2 & 1 & 2 & 4 & \\ & 4 & 4 & 3 & 4 & & \\ & & & 5 & & & \end{array}$

Figure 1

Thus the formula

$$
f_{\theta \phi}=\left(P_{0}+P_{1}+P_{2}\right) f_{00}
$$

is a second order approximation and involves the nine points numbered 0,1 and 2. On the other hand the formula

$$
f_{\theta \phi}=\left(P_{0}+P_{1}+P_{2}+P_{3}\right) f_{00}
$$

is a third order approximation and involves the 13 points numbered $0,1,2$ and 3 . If, however, at each grid point $\nabla^{2} f=g$-where $g=0$ if Laplace's equation holds$\left(\delta_{x}{ }^{2} / h^{2}+\delta_{y}{ }^{2} / k^{2}\right) f=g+$ second order terms [2], [9]. Hence $\mu_{x} \delta_{x}{ }^{3} f_{00}=$ $-\left(h^{2} / k^{2}\right) \mu_{x} \delta_{x} \delta_{y}{ }^{2} f_{00}+h^{2} \mu_{x} \delta_{x} g_{00}+$ fifth order terms, with an analogous expression for $\mu_{y} \delta_{y}{ }^{3} f_{00}$. So $P_{3} f_{00}=P_{3}{ }^{*} f_{00}+\left(h^{2} \theta_{3} \phi_{0} \mu_{x} \delta_{x}+k^{2} \theta_{0} \phi_{3} \mu_{y} \delta_{y}\right) g_{00}+$ fifth order terms, where $P_{3}{ }^{*}=\left(\theta_{2} \phi_{1}-\left(k^{2} / h^{2}\right) \theta_{0} \phi_{3}\right) \delta_{x}{ }^{2} \mu_{y} \delta_{y}+\left(\theta_{1} \phi_{2}-\left(h^{2} / k^{2}\right) \theta_{3} \phi_{0}\right) \mu_{x} \delta_{x} \delta_{y}{ }^{2}$. Thus we may replace (3) by

$$
f_{\theta \phi}=\left(P_{0}+P_{1}+P_{2}+P_{3}{ }^{*}\right) f_{00}+\left(h^{2} \theta_{3} \phi_{0} \mu_{x} \delta_{x}+k^{2} \theta_{0} \phi_{3} \mu_{y} \delta_{y}\right) g_{00} .
$$

The principal error term of (4) is the same as that of (3), i.e. $P_{4} f_{00}$ : if $f$ has continuous fourth order partial derivatives the modulus of each error term is bounded above by $(3 / 32) M_{4} \max \left(h^{4}, k^{4}\right)$. However, (4) only involves the nine points numbered 0,1 and 2 . Thus (4) has the accuracy of (3) yet uses only the same number of points as (2). This may be verified in the following example in which $f(x, y)$ is the harmonic function $\left(x+\left(x^{2}+y^{2}\right)^{1 / 2}\right)^{1 / 2}, f_{00}=f(1,1)$ and $h=k=1 / 10$. We obtain the following approximations to $f(41 / 40,41 / 40)$.

$\begin{array}{ccc}\text { Formula } & \text { Number of points } & \text { Value obtained } \\ (2) & 9 & 1.57306278 \\ (3) & 13 & 1.57307657 \\ (4) & 9 & 1.57307649\end{array}$

The correct value is 1.57307625 .

Using the same technique we find that $\mu_{x} \delta_{x}{ }^{5} f_{00}=-\left(h^{2} / k^{2}\right) \mu_{x} \delta_{x}{ }^{3} \delta_{y}{ }^{2} f_{00}+h^{2} \mu_{x} \delta_{x}{ }^{3} g_{00}$ + seventh order terms, and an analogous expression for $\mu_{y} \delta_{y}^{5} f_{00}$. Hence we may replace

$$
f_{\theta \phi}=\left(P_{0}+P_{1}+P_{2}+P_{3}+P_{4}+P_{5}\right) f_{00},
$$


which involves all 25 points of Fig. 1, by

$$
f_{\theta \phi}=\left(P_{0}+P_{1}+P_{2}+P_{3}+P_{4}+P_{5}^{*}\right) f_{00}+\left(h^{2} \theta_{5} \phi_{0} \mu_{x} \delta_{x}{ }^{3}+k^{2} \theta_{0} \phi_{5} \mu_{y} \delta_{y}{ }^{3}\right) g_{00},
$$

where

$$
\begin{aligned}
P_{5}{ }^{*}= & \theta_{4} \phi_{1} \delta_{x}{ }^{4} \mu_{y} \delta_{y}+\left(\theta_{3} \phi_{2}-\left(h^{2} / k^{2}\right) \theta_{5} \phi_{0}\right) \mu_{x} \delta_{x}{ }^{3} \delta_{y}{ }^{2} \\
& +\left(\theta_{2} \phi_{3}-\left(k^{2} / h^{2}\right) \theta_{0} \phi_{5}\right) \delta_{x}{ }^{2} \mu_{y} \delta_{y}{ }^{3}+\theta_{1} \phi_{4} \mu_{x} \delta_{x} \delta_{y}{ }^{4},
\end{aligned}
$$

which does not involve the points numbered 5. The principal error term of (6) is the same as that of (5), i.e. $P_{6} f_{00}$ : if $f$ has continuous sixth order partial derivatives, the modulus of each error term is bounded above by $(5 / 256) M_{6} \max \left(h^{6}, k^{6}\right)$.

3. Mid-Panel Formulae. The Bessel formula corresponding to (1) is

$$
f_{\theta \phi}=\sum_{r=0}^{n} Q_{r} f_{(1 / 2)(1 / 2)}
$$

where

$$
\begin{aligned}
Q_{2 s} & =\sum_{t=0}^{s} \theta_{2 s-2}^{\prime} t \phi_{2 t}^{\prime} \mu_{x} \delta_{x}{ }^{2 s-2 t} \mu_{y} \delta_{y}{ }^{2 t}+\sum_{t=1}^{s} \theta_{2 s-2 t+1}^{\prime} \phi_{2 t-1}^{\prime} \delta_{x}{ }^{2 s-2 t+1}{ }_{\delta_{y}}{ }^{2 t-1}, \\
Q_{2 s+1} & =\sum_{t=0}^{s} \theta_{2 s-2 t+1}^{\prime} \phi_{2 t}^{\prime} \delta_{x}{ }^{2 s-2 t+1} \mu_{y} \delta_{y}{ }^{2 t}+\sum_{t=0}^{s} \theta_{2 s-2 t}^{\prime} \phi_{2 t+1}^{\prime} \mu_{x} \delta_{x}{ }^{2 s-2}{ }^{2 t} \delta_{y}{ }^{2 t+1},
\end{aligned}
$$

and $\theta_{0}{ }^{\prime}=1, \quad \theta_{1}{ }^{\prime}=\theta-\frac{1}{2}, \quad \theta_{2}{ }^{\prime}=\theta(\theta-1) / 2 !, \quad \theta_{3}{ }^{\prime}=\theta\left(\theta-\frac{1}{2}\right)(\theta-1) / 3 !$, $\theta_{4}{ }^{\prime}=(\theta+1) \theta(\theta-1)(\theta-2) / 4 !, \quad \theta_{5}{ }^{\prime}=(\theta+1) \theta\left(\theta-\frac{1}{2}\right)(\theta-1)(\theta-2) / 5$ !, etc. Here it is usual to take $\left|\theta-\frac{1}{2}\right| \leqq \frac{1}{2},\left|\phi-\frac{1}{2}\right| \leqq \frac{1}{2}$. The error of (7) is approximately $Q_{n+1} f_{(1 / 2)(1 / 2)}$. Under the same conditions as previously an upper bound for the modulus of the error is $c_{n+1}^{\prime} M_{n+1}^{\prime} \max \left(h^{n+1}, k^{n+1}\right)$, where $c_{s}{ }^{\prime}=\max _{\theta, \phi} \sum_{t=0}^{s}\left|\theta_{s-t}^{\prime} \phi_{t}{ }^{\prime}\right|$ and $M_{s}{ }^{\prime}$ is an upper bound for the modulus of all the sth order partial derivatives of $f$ in the region now under consideration. If we put $n=2 m$ or $2 m+1$, according as $n$ is even or odd, then (7) involves the grid points numbered $0,1, \cdots, m$ in Fig. 2.

$$
\begin{array}{llllll} 
& & 2 & 2 & & \\
2 & 2 & 1 & 1 & 2 & \\
2 & 1 & 0 & 0 & 1 & 2 \\
& 1 & 0 & 0 & 1 & 2 \\
& 2 & 1 & 1 & 2 & \\
& & 2 & 2 & &
\end{array}
$$

FigURE 2

Thus, if we take $n=5$, we get the fifth order approximation

$$
f_{\theta \phi}=\left(Q_{0}+Q_{1}+Q_{2}+Q_{3}+Q_{4}+Q_{5}\right) f_{(1 / 2)(1 / 2)},
$$

which involves all 24 points of Fig. 2 . If, however, $\nabla^{2} f=g$, we have

$$
\left(\left(\delta_{x}{ }^{2}-\frac{1}{12} \delta_{x}{ }^{4}\right) / h^{2}+\left(\delta_{y y}{ }^{2}-\frac{1}{12} \delta_{y}{ }^{4}\right) / k^{2}\right) f=g+\text { fourth order terms }
$$

[2], [9]. Hence 


$$
\begin{aligned}
\mu_{x} \delta_{x}{ }^{4} \mu_{! l} f_{(1 / 2)(1 / 2)}= & -\frac{h^{2}}{k^{2}} \mu_{x} \delta_{x}{ }^{2} \mu_{y} \delta_{y}{ }^{2} f_{(1 / 2)(1 / 2)}+\frac{1}{12}\left(\mu_{x} \delta_{x}{ }^{6} \mu_{y}+\frac{h^{2}}{k^{2}} \mu_{x} \delta_{x}{ }^{2} \mu_{y} \delta_{y}{ }^{4}\right) f_{(1 / 2)(1 / 2)} \\
& +h^{2} \mu_{x} \delta_{x}{ }^{2} \mu_{y} g_{(1 / 2)(1 / 2)}+\text { eighth order terms }
\end{aligned}
$$

and

$$
\delta_{x}^{5} \mu_{y /} f_{(1 / 2)(1 / 2)}=-\frac{h^{2}}{k^{2}} \delta_{x}^{3} \mu_{y} \delta_{y}{ }^{2} f_{(1 / 2)(1 / 2)}+h^{2} \delta_{x}{ }^{3} \mu_{y} g_{(1 / 2)(1 / 2)}+\text { seventh order terms }
$$

with similar expressions for $\mu_{x} \mu_{y} \delta_{y}{ }^{4} f_{(1 / 2)(1 / 2)}$ and $\mu_{x} \delta_{y}{ }^{5} f_{(1 / 2)(1 / 2)}$ respectively. Thus we find that

$$
\begin{aligned}
&\left(Q_{4}+Q_{5}\right) f_{(1 / 2)(1 / 2)}=\left(Q_{4}{ }^{*}+Q_{5}{ }^{*}\right) f_{(1 / 2)(1 / 2)} \\
&+\left(h^{2} \theta_{5}{ }^{\prime} \phi_{0}{ }^{\prime} \delta_{x}{ }^{3} \mu_{y}+h^{2} \theta_{4}{ }^{\prime} \phi_{0}{ }^{\prime} \mu_{x} \delta_{x}{ }^{2} \mu_{y}+h^{2} \theta_{4}{ }^{\prime} \phi_{1}{ }^{\prime} \mu_{x} \delta_{x}{ }^{2} \delta_{y}+k^{2} \theta_{1}{ }^{\prime} \phi_{4}{ }^{\prime} \delta_{x} \mu_{y} \delta_{y}{ }^{2}\right. \\
&+ \\
&\left.+k^{2} \theta_{0}{ }^{\prime} \phi_{4}{ }^{\prime} \mu_{x} \mu_{y} \delta_{y}{ }^{2}+k^{2} \theta_{0}{ }^{\prime} \phi_{5}{ }^{\prime} \mu_{x} \delta_{y}{ }^{3}\right) g_{(1 / 2)(1 / 2)}+\text { sixth order terms }
\end{aligned}
$$

where

$$
\begin{aligned}
& Q_{4}{ }^{*}= \theta_{3}{ }^{\prime} \phi_{1}{ }^{\prime} \delta_{x}{ }^{3} \delta_{y}+\left(\theta_{2}{ }^{\prime} \phi_{2}{ }^{\prime}-\frac{h^{2}}{k^{2}} \theta_{4}{ }^{\prime} \phi_{0}{ }^{\prime}-\frac{k^{2}}{h^{2}} \theta_{0}{ }^{\prime} \phi_{4}{ }^{\prime}\right) \mu_{x} \delta_{x}{ }^{2} \mu_{y} \delta_{y}{ }^{2}+\theta_{1}{ }^{\prime} \phi_{3}{ }^{\prime} \delta_{x} \delta_{y}{ }^{3} \\
& Q_{5}{ }^{*}=\left(\theta_{3}{ }^{\prime} \phi_{2}{ }^{\prime}-\frac{h^{2}}{k^{2}} \theta_{5}{ }^{\prime} \phi_{0}{ }^{\prime}-\frac{k^{2}}{h^{2}} \theta_{1}{ }^{\prime} \phi_{4}{ }^{\prime}\right) \delta_{x}{ }^{3} \mu_{y} \delta_{y}{ }^{2} \\
&+\left(\theta_{2}{ }^{\prime} \phi_{3}{ }^{\prime}-\frac{h^{2}}{k^{2}} \theta_{4}{ }^{\prime} \phi_{1}{ }^{\prime}-\frac{k^{2}}{h^{2}} \theta_{0}{ }^{\prime} \phi_{5}{ }^{\prime}\right) \mu_{x} \delta_{x}{ }^{2} \delta_{y}{ }^{3}
\end{aligned}
$$

So, if we replace (8) by

$$
\begin{aligned}
\int_{\theta \phi}= & \left(Q_{0}+Q_{1}+Q_{2}+Q_{3}+Q_{4}{ }^{*}+Q_{5}{ }^{*}\right) f_{(1 / 2)(1 / 2)} \\
& +\left(h^{2} \theta_{5}{ }^{\prime} \phi_{0}{ }^{\prime} \delta_{x}{ }^{3} \mu_{y}+h^{2} \theta_{4}{ }^{\prime} \phi_{0}{ }^{\prime} \mu_{x} \delta_{x}{ }^{2} \mu_{y}+h^{2} \theta_{4}{ }^{\prime} \phi_{1}{ }^{\prime} \mu_{x} \delta_{x}{ }^{2} \delta_{y}\right. \\
& \left.+k^{2} \theta_{1}{ }^{\prime} \phi_{4}{ }^{\prime} \delta_{x} \mu_{y} \delta_{y}{ }^{2}+k^{2} \theta_{0}{ }^{\prime} \phi_{4}{ }^{\prime} \mu_{x} \mu_{y} \delta_{y}{ }^{2}+k^{2} \theta_{0}{ }^{\prime} \phi_{5}{ }^{\prime} \mu_{x} \delta_{y}{ }^{3}\right) g_{(1 / 2)(1 / 2)}
\end{aligned}
$$

the eight exterior values $f_{(1 / 2 \pm 5 / 2)\left(1 / 2_{ \pm 1} / 2\right)}, f_{\left(1 / 2_{ \pm 1} / 2\right)\left(1 / 2_{ \pm} / 2\right)}$ are no longer involved. The error of (9) is of the sixth order and is approximately

$$
\begin{gathered}
\left(\left(\theta_{6}{ }^{\prime} \phi_{0}{ }^{\prime}+\frac{1}{12} \theta_{4}{ }^{\prime} \phi_{0}{ }^{\prime}\right) \mu_{x} \delta_{x}{ }^{6} \mu_{y}+\theta_{5}{ }^{\prime} \phi_{1}{ }^{\prime} \delta_{x}{ }^{5} \delta_{y}+\left(\theta_{4}{ }^{\prime} \phi_{2}{ }^{\prime}+\frac{k^{2}}{12 h^{2}} \theta_{0}{ }^{\prime} \phi_{4}{ }^{\prime}\right) \mu_{x} \delta_{x}{ }^{4} \mu_{y} \delta_{y}{ }^{2}\right. \\
+\theta_{3}{ }^{\prime} \phi_{3}{ }^{\prime} \delta_{x}{ }^{3} \delta_{y}{ }^{3}+\left(\theta_{2}{ }^{\prime} \phi_{4}{ }^{\prime}+\frac{h^{2}}{12 k^{2}} \theta_{4}{ }^{\prime} \phi_{0}{ }^{\prime}\right) \mu_{x} \delta_{x}{ }^{2} \mu_{y} \delta_{y}{ }^{4}+\theta_{1}{ }^{\prime} \phi_{5}{ }^{\prime} \delta_{x} \delta_{y}{ }^{5} \\
\left.+\left(\theta_{0}{ }^{\prime} \phi_{6}{ }^{\prime}+\frac{1}{12} \theta_{0}{ }^{\prime} \phi_{4}{ }^{\prime}\right) \mu_{x} \mu_{y} \delta_{y}{ }^{6}\right) f_{(1 / 2)(1 / 2)} .
\end{gathered}
$$

If $f$ has continuous sixth order partial derivatives, an upper bound for the modulus of the error is

$$
\left(\frac{1}{64}+\frac{1}{512}\left(\frac{h}{k}+\frac{k}{h}\right)^{2}\right) M_{6}^{\prime} \max \left(h^{6}, k^{6}\right) \text {. }
$$

4. Cubature Formulae. Cubature formulae for integrating over a region throughout which Poisson's equation holds may be developed in a similar way. As a simple 
illustration, let us consider mid-point formulae to evaluate $\int_{-1}^{1} \int_{-1}^{1} f_{\theta \phi} d \theta d \phi$, assuming that $\nabla^{2} f=g$. By integrating (1), we get

$$
\int_{-1}^{1} \int_{-1}^{1} f_{\theta \phi} d \theta d \phi=\sum_{r=0}^{m} \int_{-1}^{1} \int_{-1}^{1} P_{2 r} d \theta d \phi f_{00}
$$

with an error of the $(2 m+2)$ th order of approximately $\int_{-1}^{1} \int_{-1}^{1} P_{2 m+2} d \theta d \phi f_{00}$. If $f$ has continuous $(2 m+2)$ th order partial derivatives, an upper bound for the modulus of the error is $d_{m+1} M_{2 m+2} \max \left(h^{2 m+2}, k^{2 m+2}\right)$, where

$$
d_{s}=\sum_{t=0}^{s}\left|\int_{-1}^{1} \int_{-1}^{1} \theta_{2 s-2 t} \phi_{2 t} d \theta d \phi\right|
$$

Thus, if we take $m=2$, we get

(10) $\int_{-1}^{1} \int_{-1}^{1} f_{\theta \phi} d \theta d \phi=\left(4+\frac{2}{3} \delta_{x}{ }^{2}+\frac{2}{3} \delta_{y}{ }^{2}-\frac{1}{45} \delta_{x}{ }^{4}+\frac{1}{9} \delta_{x}{ }^{2} \delta_{y}{ }^{2}-\frac{1}{45} \delta_{y}{ }^{4}\right) f_{00}$,

with a sixth order error of approximately

$$
\frac{1}{1890}\left(5 \delta_{x}{ }^{6}-7 \delta_{x}{ }^{4} \delta_{y}{ }^{2}-7 \delta_{x}{ }^{2} \delta_{y}{ }^{4}+5 \delta_{y}{ }^{6}\right) f_{00} .
$$

If $f$ has continuous sixth order partial derivatives, the above upper bound for the modulus of the error is $(4 / 315) M_{6} \max \left(h^{6}, k^{6}\right)$. This formula involves the points numbered $0,1,2$ and 3 in Fig. 1. We note that the points numbered 3 are outside the region of integration. To get a formula which has a sixth order error and only involves the points numbered 0,1 , and 2 , we may use the fact that $\nabla^{2} f=g$, which implies that

$$
\delta_{x}{ }^{4} f_{00}=-\frac{h^{2}}{k^{2}} \delta_{x}{ }^{2} \delta_{y}{ }^{2} f_{00}+\frac{1}{12}\left(\delta_{x}{ }^{6}+\frac{h^{2}}{k^{2}} \delta_{x}{ }^{2} \delta_{y}{ }^{4}\right) f_{00}+h^{2} \delta_{x}{ }^{2} g_{00}+\text { eighth order terms },
$$

with a similar expression for $\delta_{y}{ }^{4} f_{00}$, and thus replace (10) by

$$
\begin{aligned}
\int_{-1}^{1} \int_{-1}^{1} f_{\theta \phi} d \theta d \phi= & \left(4+\frac{2}{3} \delta_{x}{ }^{2}+\frac{2}{3} \delta_{y}{ }^{2}+\left(\frac{1}{9}+\frac{h^{2}}{45 k^{2}}+\frac{k^{2}}{45 h^{2}}\right) \delta_{x}{ }^{2} \delta_{y}{ }^{2}\right) f_{00} \\
& -\frac{1}{45}\left(h^{2} \delta_{x}{ }^{2}+k^{2} \delta_{y}{ }^{2}\right) g_{00} .
\end{aligned}
$$

The resulting error is approximately

$$
\frac{1}{3780}\left(3 \delta_{x}{ }^{6}-7\left(2+\frac{k^{2}}{h^{2}}\right) \delta_{x}{ }^{4} \delta_{y}{ }^{2}-7\left(2+\frac{h^{2}}{k^{2}}\right) \delta_{x}{ }^{2} \delta_{y}{ }^{4}+3 \delta_{y}{ }^{6}\right) f_{00} .
$$

Under the same conditions as previously, the above upper bound for the molulus of the error is

$$
\left(\frac{1}{189}+\frac{1}{540}\left(\frac{h}{k}+\frac{k}{h}\right)^{2}\right) M_{6} \max \left(h^{6}, k^{6}\right) .
$$

The special case of (11) in which $h=k$ has been considered by Bickley [8].

5. Acknowledgement. The author is indebted to a referee for suggesting the allusion to Collatz' work and some clarification. 
Department of Mathematics

King's College

London, W.C. 2, England

1. K. Pearson, Tracts for Computers. III: On the Construction of Tables and on Interpolationpart II: Bi-Variate Tables, London, 1920.

2. K. Ho, "Double interpolation formulae and partial derivatives in terms of finite differences," $M T A C$, v. 9, 1955 , pp. 52-62. MR 17, 538 .

3. R. A. Buckingham, Numerical Methods, 2nd ed., London, 1962.

4. L. Collatz, The Numerical Treatment of Differential Equations, 3rd ed., Springer-Verlag, Berlin, 1960. MR $22 \# 322$.

5. E. J. NyströM, "Zur numerischen Lösung von Randwertaufgaben bei gewöhnlichen Differentialgleichungen," Acta Math., v. 76, 1945, pp. 157-184. MR 7, 220.

6. G. STRACKe, Bahnbestimmung der Planeten und Kometen, Berlin, 1929.

7. L. V. Kantorovich \& V. I. Krylov, Approximate Methods of Higher Analysis, Interscience, New York; Noordhoff, Groningen, 1958.

8. W. G. BickLey, "Finite difference formulae for the square lattice," Quart. J. Mech. Appl. Math., v. 1, 1948, pp. 35-42. MR 9, 623 .

9. D. J. PanOw, Formelsammlung zur numerischen Behandlung partieller Differentialgleichungen nach dem Differenzenverfahren, Akademie-Verlag, Berlin, 1955. MR 17, 196. 